\title{
Complete removal as a routine treatment for any cardiovascular implantable electronic device-associated infection
}

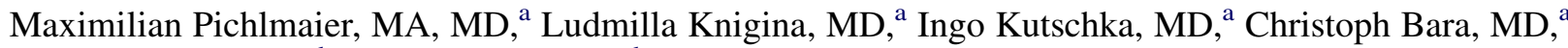 \\ Hanno Oswald, MD, ${ }^{b}$ Gunnar Klein, MD, ${ }^{b}$ Theodosius Bisdas, MD, ${ }^{a}$ and Axel Haverich, MD, PhD ${ }^{a}$
}

\begin{abstract}
Objective: Pacemaker and implantable cardioverter defibrillator lead endocarditis mandates removal of all foreign material. In supposedly limited (pocket) infections, such a radical approach is still controversial. Thus, some patients are potentially exposed to persistent and recurrent infection because of retained material. Procedural risks and the success of eradicating infection were examined if involvement of the complete system was assumed in any cardiovascular implantable electronic device infection and complete removal was thus mandatory.
\end{abstract}

\begin{abstract}
Methods: A 12-year experience with 192 consecutive cases of bacterial pacemaker (152) or defibrillator (40) infections is presented. Complete removal of all prosthetic material was always aimed for. This was followed by antibiotic treatment for 4 to 6 weeks under temporary pacing if required, and then the new system was implanted. A total of 104 parameters concerning patient characteristics and operative and postoperative treatment were examined for their influence on outcome.

Results: Infection was eradicated in $92.8 \%$ of patients. Recurrence was predominantly caused by failure to remove all prosthetic material $(P<.001)$. If the protocol was strictly followed, infection was eradicated in $97.4 \%$ of patients. Conversely, $71.4 \%$ of patients with retained material showed recurrence. Further risk factors were poor dental hygiene and evidence of chronic subclinical infection. Morbidity and mortality of the interventional and open procedures were low. Open lead extraction was performed primarily in 34 patients $(17.7 \%)$ and secondarily in 3 patients $(1.9 \%)$. Temporary pacing and long-term antibiotic treatment were well tolerated.

Conclusions: Complete removal of prosthetic material in any cardiovascular implantable electronic device infection is safe and associated with low morbidity and mortality. Success of eradicating infection is high if all system components are removed. Temporary pacing in dependent patients may be performed safely on an outpatient basis. (J Thorac Cardiovasc Surg 2011;142:1482-90)
\end{abstract}

The incidence of bacterial infections of cardiac pacemakers and defibrillators (cardiovascular implantable electronic devices [CIEDs]) has increased ${ }^{1,2}$ independently of changing patient characteristics and the growing relative proportion of implantable cardioverter defibrillator (ICD) recipients. ${ }^{1}$ This continuing trend requires rigorous management strategies. ${ }^{3-5}$ Risk factors for the development of CIED infection have repeatedly been identified. ${ }^{5-13}$ Renal failure, ${ }^{6,14,15}$ diabetes and congestive heart failure, ${ }^{6,14}$ the number of previous operations, ${ }^{2,6,14,16}$ increasing number of leads, ${ }^{16}$ indwelling central venous lines, ${ }^{16}$ experienced bacteremias, ${ }^{17}$ and thrombosis on the leads ${ }^{10,18}$ all predispose to later infection, whereas steroid treatment

From the Department of Cardiac, Thoracic, Transplantation, and Vascular Surgery, ${ }^{\mathrm{a}}$ and Department of Cardiology and Angiology, ${ }^{\mathrm{b}}$ Hannover Medical School, Hannover, Germany.

Funding provided by departmental research funds.

Disclosures: Authors have nothing to disclose with regard to commercial support.

Received for publication Jan 19, 2010; revisions received Oct 17, 2010; accepted for publication Nov 2, 2010; available ahead of print May 13, 2011.

Address for reprints: Maximilian Pichlmaier, MA, MD, Cardiac, Thoracic, Transplantation, and Vascular Surgery, Hannover Medical School, D-30925 Hannover, Germany (E-mail: pichlmaier.maximilian@mh-hannover.de).

$0022-5223 / \$ 36.00$

Copyright (c) 2011 by The American Association for Thoracic Surgery doi:10.1016/j.jtcvs.2010.11.059 remains controversial. ${ }^{15}$ The experience of the physician performing the procedure and the circumstances of implantation (eg, catheter laboratory vs operating room) have been discussed, ${ }^{19}$ but conclusive data are lacking. Current focus is on prevention by avoidance of known risk factors and prophylactic perioperative antibiotics ${ }^{13}$ (metaanalysis ${ }^{20}$ ) on the one hand and optimized treatment of established infection on the other.

For the treatment of proven septicemia or lead endocarditis, there is sufficient evidence to support obligatory removal of all prosthetic material under continued antibiotic therapy ${ }^{6,18,19,21-26}$ (North American Society of Pacing and Electrophysiology/Heart Rhythm Society). ${ }^{11,19}$ Antibiotic treatment alone has been abandoned in this context. ${ }^{7,19,22,25}$ However, removal of system components with, eg, the leads left in place supposedly isolated pocket infections, although repeatedly criticized, ${ }^{9,25,27}$ is still performed, and current recommendations do not categorically object to this. ${ }^{19}$ There also has been a revival of intermediate procedures with irrigation and muscle flaps as an alternative to complete removal. ${ }^{28}$ The definition of limited infection, especially in the absence of diagnostic tools, creates the dilemma. ${ }^{29,30}$ An additional dispute is about whether to implant the new permanent system at the same time or after a period of temporary 


\section{Abbreviations and Acronyms \\ CIED = cardiovascular implantable electronic device \\ $\mathrm{CRP}=$ C-reactive protein \\ ESR = erythrocyte sedimentation rate \\ ICD = implantable cardioverter defibrillator \\ MRSA $=$ methicillin-resistant Staphylococcus aureus}

pacing and antibiotic treatment. ${ }^{2,4,21,26,31,32}$ In pacemakerdependent patients and patients with class I indications for ICD treatment, this is a management and cost issue.

In 1996, the current authors began to treat consecutive patients with any CIED infection as if there was always lead involvement, and thus complete removal of all prosthetic material was mandatory in every case. The protocol included 3 to 6 weeks of antibiotic treatment and temporary pacing (if necessary) between extraction and reimplantation, as others have since advocated. ${ }^{31,32}$ The retrospective analysis of this prospectively collected data set was performed to examine if such a rigorous approach achieves adequate results with acceptable morbidity and mortality without the problem of having to distinguish between various forms of CIED infection.

\section{MATERIALS AND METHODS \\ Patients and Preoperative Investigations}

Patients treated from 1996 to 2007 for CIED infection were included after giving informed consent (Declaration of Helsinki) for the use of their data. The diagnosis was based on local changes of the device pocket, C-reactive protein (CRP), erythrocyte sedimentation rate (ESR), and the Duke criteria for endocarditis. ${ }^{4,6,26}$ Recurrent device dislocations where 1 or more corrective procedures had been performed qualified as chronic infection once obvious causes had been excluded. Vegetations were primarily treated with intravenous antibiotics and anticoagulation to reduce size and microbial load. Thereafter, vegetations larger than $1 \mathrm{~cm}$ or definite valvular involvement led primarily to open surgery (Figure 1).

Devices were interrogated to estimate the degree of dependence and intrinsic rhythm challenged by programming to ventricular demand mode (VVI) 30 beats/min, including the use of sympathomimetic drugs. If less than $10 \%$ pacing per 24 hours was documented, temporary pacing was not primarily planned in these patients. Stratification into therapy regimens followed (Figure 1).

\section{Surgical Procedures}

All procedures were performed by a cardiovascular surgeon in an operating room using a conventional mobile $\mathrm{C}$-arm. Pacemaker-dependent patients received a temporary transcutaneous transvenous pacing system, which has since been described by others. ${ }^{31,32}$ Bipolar active fixation leads (Medtronic Inc. [Minneapolis, Minn] 4076/5076, 58 or $65 \mathrm{~cm}$ ) were introduced preferably ipsilateral to the infected device via the subclavian/jugular vein, leaving the contralateral side for implantation of the permanent device. Exceptions were made if the inflammatory response around the device pocket was large and could not be circumvented with the temporary lead. After endocardial positioning, the proximal part of the lead was tunneled subcutaneously 15 to $25 \mathrm{~cm}$ parallel to the sternum and externalized. The lead was secured on the deep fascia at both sites using nonresorbable sutures. A pacemaker programmed to bipolar function was connected externally to the lead. Extensive debridement (all scar tissue and fibrous capsule) followed at the site of the device pocket. Swabs were taken for microbiological workup. Removal of leads was achieved using Byrd dilator sheaths (Cook Medical, Bloomington, Ind) in combination with locking stylets (VascoExtor, VascoMed, Binzen, Germany, or Spectranetics, Colorado Springs, Colo) when necessary. Proximal adhesions were primarily addressed with passive sheaths alone. The regular positioning stylet was exchanged for a locking stylet if stabilization became insufficient or distal adhesions became apparent. Locking stylets were primarily used without sheaths if only distal adhesions were encountered. Electrosurgical sheaths (Cook Medical) have lately (since 2005) facilitated extraction in cases of severe adhesions. The lead extraction approach was always along the implant vein. Other approaches were not used in infection. Extraction was thought to be safer and thus approached more forcefully if patients had undergone previous conventional cardiac surgery. Development of effusions is less common because of the adhesions, and the alternative open procedure carries a substantially higher risk.

Open surgery was performed through a median sternotomy with cardiopulmonary bypass using cardioplegic arrest as required for concomitant procedures. The right atrium was opened, and the damage of the tricuspid valve was assessed; if required, repair was attempted whenever possible. The leads were cut in the superior vena cava, and the distal portion was explanted. The device pocket and proximal lead segments were attended to at the end after closure of the sternotomy. Extraction sheaths were used as required to free the lead remnant from adhesions under the clavicle (1 case). Temporary pacing was achieved via epicardial temporary pacing wires. Some patients received epicardial permanent pacing wires at the digression of the surgeon. Electrode tips underwent microbiological evaluation applying an extended endocarditis protocol.

Primary wound closure of the device pocket was always attempted using resorbable monofilament sutures for both subcutaneous fascia and skin. This followed radical resection of the complete device pocket and all associated scar tissue, including the muscle fascia. Suction drains were placed and left for 5 days. Antibiotic treatment was not started until the time of operation except in sepsis or if patients were already receiving endocarditis treatment (vegetations) to increase the yield of microbiological examination of the intraoperative samples. A broad-spectrum cephalosporin was given, and the treatment was adjusted according to susceptibility. Intravenous administration was changed to oral, excluding patients with valvular endocarditis, after 3 to 7 days. Antibiotic treatment was continued for 3 to 6 weeks depending on the microbiological culture results (positive lead tip 6 weeks, negative lead tip 3 weeks). These comparatively long intervals were chosen in 1996 with reference to the treatment of valvular endocarditis before surgical valve replacement (6 weeks minimum). If microbiological culture from the leads remained negative, the interval was empirically halved to avoid potential complications of prolonged antibiotic therapy. Patients with a pacemaker were discharged if possible; patients with an ICD more often remained as inpatients on telemetry. Currently, patients with no shock history and with a primarily prophylactic indication are also discharged. In the absence of systemic infection (ESR/CRP), patients then received a new permanent pacemaker/ICD on the contralateral side. Antibiotic treatment was continued for a further 2 to 3 weeks. Follow-up was at 6 weeks. With the use of questionnaires, long-term follow-up was performed by telephone interview of the patient, family practitioner, and cardiologist.

\section{Study Design and Statistics}

Analyses and graphs were performed using MedCalc2.4.0 (Marienkerke, Belgium). Categoric variables are presented as percentages, and 


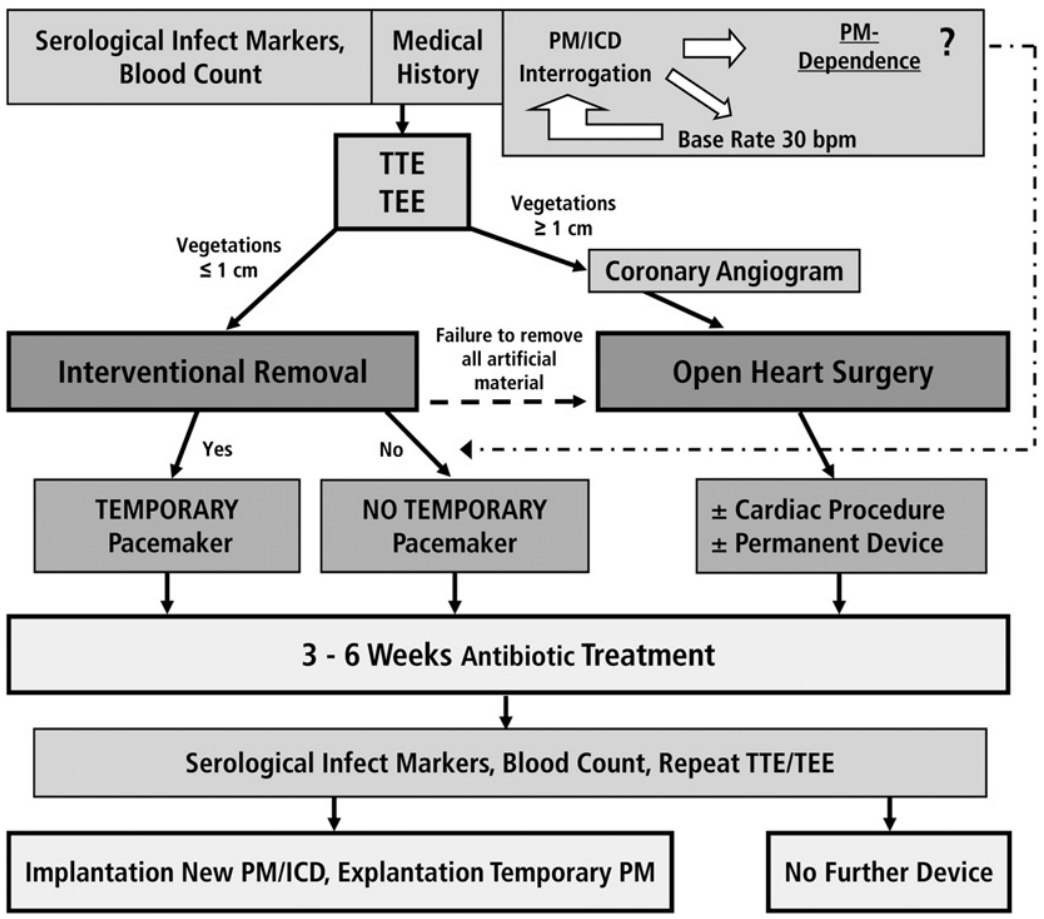

FIGURE 1. Treatment protocol with stratification of patients into interventional removal or open surgery. PM, Pacemaker; $I C D$, implantable cardioverter defibrillator; TTE, transthoracic echocardiography; TEE, transesophageal echocardiography.

continuous variables are presented as means ( \pm standard deviation) or medians (ranges) as appropriate (Kolmogorov-Smirnov test). Categoric data were examined with Pearson's chi-square test. Normally distributed continuous variables were compared with the $t$ test for independent variables, and not-normally distributed data were compared with the Mann-Whitney test. Homogeneity of variances was investigated by Levine's test. Freedom from recurrence of infection was analyzed using the Kaplan-Meier method (log-rank test).

The primary end point was the failure to eradicate infection ("recurrence"). A total of 104 parameters relating to patient characteristics, presenting clinical picture, surgical details, and postoperative course were analyzed for their relevance. Mortality, short- and medium-term morbidity, technical aspects of the surgery, and acceptance of the treatment protocol were secondarily evaluated. A Cox proportional hazard model including all parameters (pre-, intra-, and postoperative) was used to estimate relative risk for recurrence of infection. Parameters with less than 10 events were excluded from the proportional hazard regression model.

\section{RESULTS \\ Patient Characteristics}

From November 1996 to July 2007, 3491 surgical procedures involving CIEDs were performed. Infection was preoperatively diagnosed in 178 patients (mean age 69 years, $27 \%$ were female) who underwent 192 procedures. Thus, 12 patients underwent 2 or 3 procedures (Figure 2). The majority of patients (119) were referred from other hospitals, 35 with recurrent/persistent infection. One third of patients (57) had undergone surgical procedures in which old leads had been abandoned. In 124 patients (69.7\%), there had been 2 (1-5) surgical interventions on the pocket since implantation. In $32.3 \%$ of patients, at least 1 surgical intervention was to deal with complications rather than battery depletion $(67.7 \%)$.

In 133 procedures $(69.3 \%)$, an isolated pocket problem was suspected (53 showed purulent secretion). Pocket revisions elsewhere had preceded presentation in 65 patients, and 15 patients exhibited a minor problem, such as persistent pain, skin irritation, or recurrent dislocation of the device with secondary confirmation of infection on microbiological workup. The remaining 59 patients were referred with lead endocarditis secured echocardiographically (51) or by blood cultures (53). Vegetations varied from 0.5 to 4 $\mathrm{cm}$. Tricuspid insufficiency was found in $25 \%$ of patients. Only 3 patients had isolated serologic or clinical evidence of some form of ongoing systemic infection without a secured diagnosis.

Retrospectively, CRP/ESR only marginally influenced surgical decision-making at the stage of primary admission. Thus, elevated levels were observed in cases of obvious lead endocarditis or acute postoperative pocket infections but were absent in cases of recurrent device dislocations.

\section{Procedures}

Details of the procedures performed are shown in Table 1 and Figure 2. All open procedures were primarily performed 

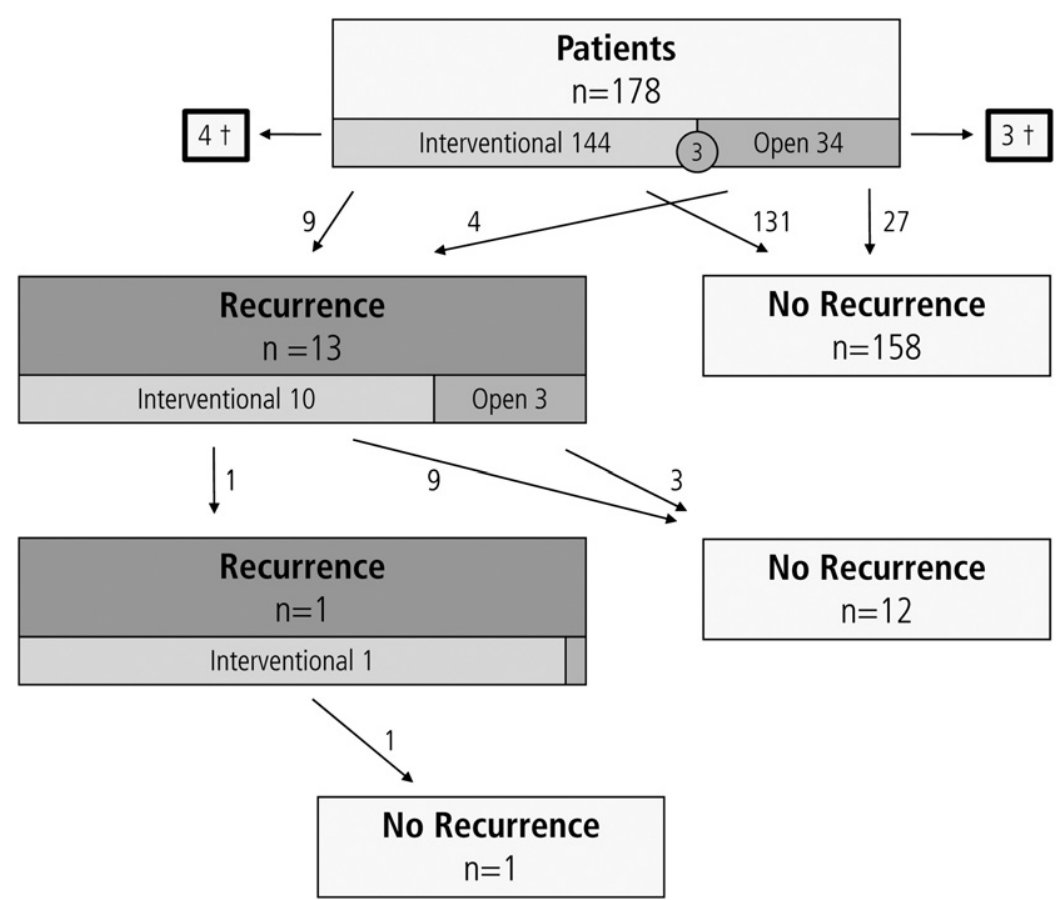

FIGURE 2. Treatment pathways and outcome of patients. Three patients (in circle) crossed over primarily from interventional removal (number of procedures) to open surgery (number of procedures) because of retained material.

for CIED infection. The high incidence of concomitant procedures in this group reflects patient comorbidity and the policy to attend to correctable cardiac problems if bypass is required for lead removal whenever possible.

Complete removal of all prosthetic material was primarily achieved in 165 patients $(92.7 \%)$. Three patients proceeded directly to secondary open procedures because of failure to remove all material interventionally (Figure 2). Retained material in addition to leads included remnants of the lead insulation. Open operations also led to secondary procedures. In 3 cases, lead remnants were inadvertently left in the access vein proximally.

In 190 cases (99\%), primary wound closure was possible, including 4 rotational skin flaps. In all these instances, the wound healed completely without the need for further intervention. Evidence for ongoing soft tissue infection or undue secretions via the drainage canal led to operative revisions in 2 cases (Table 1).

Implantation of the new permanent device followed after a median time interval of $6(1-47)$ weeks. Long interim periods arose where no new permanent device was implanted because the indication was questionable, but later the patient required a pacemaker again. Elevated CRP/ESR at this stage led to a delay of reimplantation.

\section{Outcome}

Follow-up was 55 (24-156) months with an obligatory minimum of 24 months.
Mortality. Thirty-three patients died (CIED-related in 7, cardiac reasons in 12, other reasons in 14). Of the 7 $(3.9 \%)$ CIED infection-related deaths, $3(1.7 \%)$ followed open operations within 30 days because of ongoing valvular endocarditis or the operation (Figure 2). Four patients died after interventional procedures (Figure 2), 2 within 30 days and 2 later. The 2 early deaths after successful transvenous removal of all material were due to ongoing uncontrolled endocarditis (methicillin-resistant Staphylococcus aureus [MRSA] in 1 and extensive 3-valve involvement in 1). No patient died after a secondary open operation after a failed transvenous extraction. The cause of death is not known in 2 patients, but deaths occurred more than 2 years after surgery.

Recurrence of infection. Fourteen cases $(7.8 \%)$ of recurrence were observed (Figure 2). They presented 1 to 14 months after surgery with signs of infection, vegetations on the remaining/new leads, or tricuspid valve endocarditis, and reentered the treatment protocol accordingly (1 patient twice).

The single most significant, independent parameter for recurrence was incomplete removal of prosthetic material in 10 cases $71.4 \%$ (Figure 3). Reasons for failure to immediately perform another procedure in these patients to satisfy the protocol were (1) leaving the leads in patients with primary pocket problems and very high risk for the removal procedure (5 patients); (2) leaving torn leads in patients with high risk (2 patients); and (3) leaving intravenous 
TABLE 1. Procedures performed and general details

\begin{tabular}{|c|c|c|}
\hline Parameter & No. & Fraction/range \\
\hline \multicolumn{3}{|l|}{ Open surgery $(\mathrm{n}=37)$} \\
\hline Operation time & $165 \mathrm{~min}$ & $36-600 \mathrm{~min}$ \\
\hline Bypass time & $65 \mathrm{~min}$ & $15-161 \mathrm{~min}$ \\
\hline Aortic clamp time & $39 \min$ & $5-119 \min$ \\
\hline Postoperative ventilation & $11.5 \mathrm{~h}$ & $3-564 \mathrm{~h}$ \\
\hline Hospital stay & $16.1 \mathrm{~d}$ & $9-31 \mathrm{~d}$ \\
\hline Additional cardiac procedures & $\begin{array}{l}\text { TVR } 41.6^{\circ} \\
\text { closure } 8.3^{\circ} \\
4.2 \%, \text { com }\end{array}$ & $\begin{array}{l}\text { AVR } 16.7 \%, \text { ASD } \\
\text { MVR } 4.2 \%, \text { CABG } \\
\text { tions thereof } 25 \%\end{array}$ \\
\hline \multicolumn{3}{|l|}{ Interventional procedures $(\mathrm{n}=155)$ : } \\
\hline General anesthesia & 133 & $85.8 \%$ \\
\hline Procedure time & $60 \mathrm{~min}$ & $12-240 \mathrm{~min}$ \\
\hline $\mathrm{X}$-ray exposure & $4.8 \mathrm{~min}$ & $0-43 \mathrm{~min}$ \\
\hline Age of leads & \multicolumn{2}{|c|}{$(23 \%<1$ y implanted $)$} \\
\hline Locking stylets (procedures) & 75 & $48 \%$ \\
\hline Dilator sheaths (procedures) & 52 & $34 \%$ \\
\hline \multicolumn{3}{|l|}{ Morbidity: } \\
\hline Valve damage requiring TVR & 2 & $1 \%$ \\
\hline $\begin{array}{l}\text { Soft tissue infection } \\
\text { requiring surgery }\end{array}$ & 2 & $1 \%$ \\
\hline Hematoma requiring surgery & 9 & $4.7 \%$ \\
\hline Hematoma not requiring surgery & 9 & $4.7 \%$ \\
\hline $\begin{array}{l}\text { Pericardial effusion not } \\
\text { requiring drainage }\end{array}$ & 4 & $2.1 \%$ \\
\hline Access vein thrombosis & 3 & $1.6 \%$ \\
\hline
\end{tabular}

lead fragments during an open operation for endocarditis (3 patients). These eventually led to further surgery (interventional in 8 patients, open in 2 patients) with complete removal of retained material, and infection then resolved (Figure 2).

Because of refusal to consent (2 patients) or excessive procedural risk ( 2 patients), a further 4 patients did not have all prosthetic material removed. Only 2 patients remain without symptoms (at 29 and 69 months), and 2 patients have died of sepsis at 1.5 and 84 months, although they initially presented with a presumed isolated pocket infection (Figure 3).

Three secondary interventional procedures and 1 open operation were necessary to deal with recurrence despite primary successful complete removal of all prosthetic material $(2.4 \%)$ (Figure 3$)$. Two of these $(50 \%)$ followed supposedly isolated pocket infections. The overall secondary success rate of eradication of infection once all prosthetic material was removed was $97.4 \%$.

Factors associated with failure to eradicate infection are shown in Table 2. Two parameters (incomplete removal of artificial material 10/4 and cessation of CIED therapy
0/14) had to be excluded from the Cox model because there were less than 10 events in one of the groups. They were evaluated using the Kaplan-Meier method. Conversely, Table 3 shows a selection of parameters that did not reach significance but might have been expected to. Data completeness for the set of 104 parameters was $99.1 \%$.

A recurrence of infection or late lead failure did not develop in any of the 25 patients who received permanent epicardial leads during an open procedure. Figure 4 shows an example of a failed interventional lead removal after an attempt at extraction at another institution, and eventually an open procedure had to be performed.

Morbidity of the procedures. Cardiac tamponade or venous tears did not occur. Further complications are summarized in Table 1.

Patients' acceptance. The hospital stay for patients with a pacemaker was 7 (2-71) days primarily because drains are not removed before postoperative day 5 and the microbiological workup may take 7 days. Patients with an ICD stayed in the hospital for 12 (7-46) days. In the majority of patients, the procedure was perceived acceptable in terms of stress, length of hospital stay, and burden of the postoperative antibiotic therapy. In $12 \%$ of patients, the patient or the treating physician/cardiologist reported minor difficulties with the temporary pacing or antibiotic regimen. In 9 patients $(4.7 \%)$, the antibiotic was changed for diarrhea (2), renal insufficiency (2), and skin rash or minor side effects (5). Reprogramming of the temporary devices was necessary in 9 patients (pacemaker syndrome in 1 and chronotropic insufficiency in 8). No case of new atrial fibrillation was observed. There was no dislodgement, dysfunction, or wound problem at the percutaneous passage of the temporary lead.

\section{Microbiology}

In $81.8 \%$ of cases, bacteria were identified in culture. Coagulase-negative staphylococci (epidermidis, capitis, haemolyticus) were the most common organisms (54.7\%) followed by Staphylococcus aureus (27.6\%) and grampositive rods. MRSA was observed in 3 patients. Of the 25 patients in whom no definitive diagnosis of device infection was made preoperatively, $80.6 \%$ returned a positive culture result from intraoperative samples, predominantly Staphylococcus epidermidis. Failure to eradicate infection was not obviously associated with the type or presence of organisms, and recurrent disease always yielded the same organism.

\section{DISCUSSION}

The distinction between isolated pocket and complete device/lead involvement in bacterial CIED infection can be difficult. ${ }^{27}$ However, treatment strategies depend on this differentiation, and complete removal of all prosthetic material is only recommended for lead endocarditis. ${ }^{8,13,19}$ To avoid 


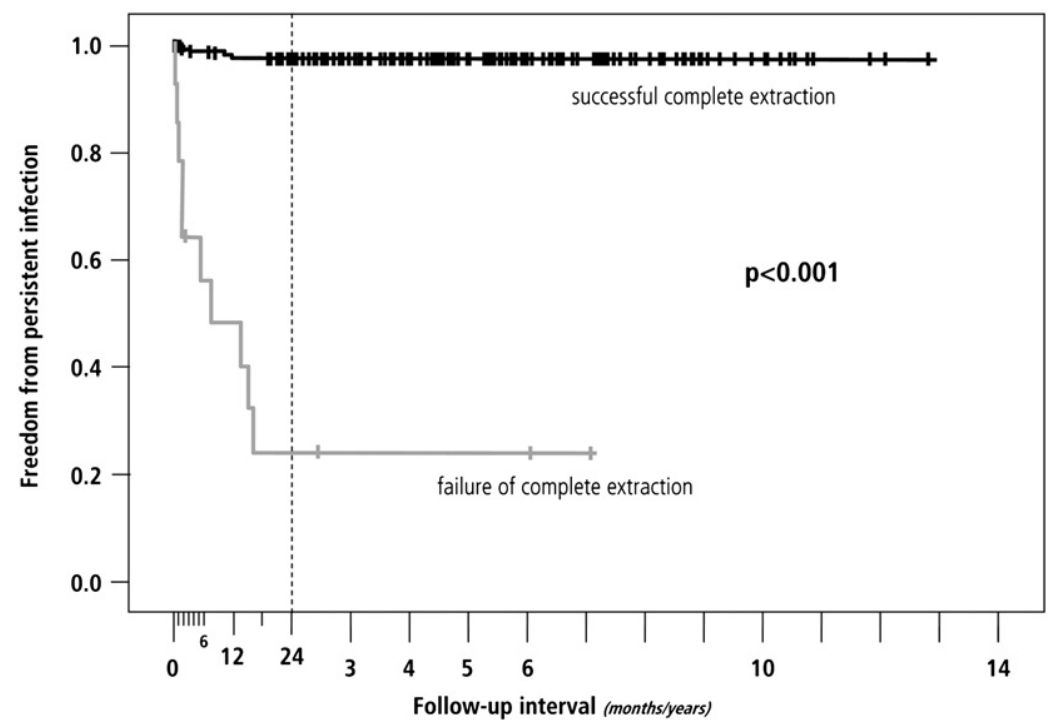

FIGURE 3. Kaplan-Meier plot of freedom from infection dependent on complete removal of prosthetic material. Note nonlinear time axis stressing the initial 24 months after surgery.

recurrences after partial removal of CIED components, ${ }^{6,27}$ the results of a radical policy of mandatory complete removal of all prosthetic material in all cases were examined. The results confirm acceptable mortality and morbidity, and the recurrence rate of $2.6 \%$ is low. The main criticism concerning this strategy to treat any CIED infection is that a potential number of patients with no or limited infection of their device components undergo extensive, radical therapy. This is contrasted by the deleterious result of patients returning with smoldering infections on previously abandoned components. ${ }^{6,27}$ The appropriateness of this approach may be judged after a detailed analysis of the complication and success rates in comparison with published data. A prospective trial could elucidate this issue but is applicable only to patients with "mild" disease.

\section{Complications}

The protocol has not caused unduly high complication rates. Mortality resulted from device infection or open operation in $3.9 \%$ and transvenous removal procedures in $2.2 \%$ (not procedure related). The significance of CIED infection in causing mortality has been demonstrated, ${ }^{2,6}$ but others have reported a higher procedural mortality. ${ }^{33}$ This may be by chance given the heterogeneity of these patients or may relate to a lower threshold in patients with lead endocarditis primarily undergoing an open procedure, which naturally carries a higher risk. The opposite is found, however, in that the proportion of open procedures in this series is higher than in other reports. ${ }^{6,26,34,35}$ This may be explained by a higher incidence of valvular involvement or concomitant cardiac disease in this patient population who were all referred to a surgical unit. Furthermore, the

TABLE 2. Failure to eradicate infection: Significant parameters

\begin{tabular}{|c|c|c|c|c|}
\hline \multirow[b]{2}{*}{ Parameter } & \multirow{2}{*}{$\begin{array}{c}\text { Chi-square } \\
P \text { value } \\
\end{array}$} & \multicolumn{3}{|c|}{ Cox regression } \\
\hline & & Hazard ratio & $95 \% \mathrm{CI}$ & $P$ value \\
\hline \multicolumn{5}{|l|}{ Preoperative } \\
\hline Previous lead dislocation & .04 & 3.62 & $1.10-11.88$ & .03 \\
\hline Imminent cutaneous perforation & .02 & 9.79 & $2.38-40.23$ & .001 \\
\hline Poor dental hygiene & .04 & 4.25 & $1.12-16.04$ & .003 \\
\hline \multicolumn{5}{|l|}{ Intraoperative } \\
\hline Interventional removal & .005 & 0.30 & $0.10-0.84$ & .002 \\
\hline Incomplete removal of prosthetic material & $<.001$ & \multicolumn{2}{|c|}{ Kaplan-Meier (Figure 2) } & $<.001 *$ \\
\hline \multicolumn{5}{|l|}{ Postoperative } \\
\hline Cessation of CIED therapy & .03 & $(<1.00)$ & Kaplan-Meier & $.01 *$ \\
\hline Contralateral implantation of permanent device & .02 & 0.23 & $0.07-0.76$ & .01 \\
\hline
\end{tabular}

$C I$, Confidence interval; $C I E D$, cardiovascular implantable electronic device. Prognostic covariates for recurrence of infection as identified by chi-square test and subsequent multivariate Cox regression analysis. *Incomplete removal of prosthetic material and cessation of CIED therapy were assessed by log-rank test (Kaplan-Meier) as explained in the text. 
TABLE 3. Failure to eradicate infection: Parameters of no significance

\begin{tabular}{|c|c|}
\hline Parameter & $P$ value \\
\hline \multicolumn{2}{|l|}{ Preoperative } \\
\hline Age & .45 \\
\hline $\mathrm{ICD} /$ pacer & .27 \\
\hline Latest revision in our hospital & .91 \\
\hline No. of previous operations on the pocket & $.79^{*}$ \\
\hline Age of leads & .48 \\
\hline Infection follows primary implantation & .79 \\
\hline Infection follows battery replacement & .83 \\
\hline Local infection (pocket) & .90 \\
\hline Systemic infection (leads) & .52 \\
\hline Combined (pocket and leads) & .71 \\
\hline Positive inflammatory markers & .91 \\
\hline Positive blood culture & 69 \\
\hline Cutaneous perforation & .97 \\
\hline Purulence & .10 \\
\hline Positive microbiology from leads & .77 \\
\hline MRSA & .85 \\
\hline Severe immunosuppression & .44 \\
\hline Cortisone therapy & .81 \\
\hline Renal failure & .38 \\
\hline Diabetes mellitus & .76 \\
\hline ASA score & $.55^{*}$ \\
\hline \multicolumn{2}{|l|}{ Intraoperative } \\
\hline Use of extraction stylets & $.13 \dagger$ \\
\hline Epicardial temporary pacing & $.31 \dagger$ \\
\hline Simultaneous epicardial permanent device & $.71 \dagger$ \\
\hline Valvular endocarditis & $.60 \dagger$ \\
\hline Delayed permanent device & .46 \\
\hline Ipsilateral permanent device & .40 \\
\hline Open explantation & .57 \\
\hline Tricuspid reconstruction & $.89 \dagger$ \\
\hline \multicolumn{2}{|l|}{ Postoperative } \\
\hline Temporary pacing & .85 \\
\hline Duration antibiotic treatment & .63 \\
\hline No permanent device & .72 \\
\hline
\end{tabular}

protocol dictated a primarily open procedure for patients with vegetations greater than $1 \mathrm{~cm}$. The authors changed this policy from 2008 (no patients included thereafter) so that vegetations up to $2 \mathrm{~cm}$ are now accepted for interventional removal; this seems safe and is reflected in the most recent recommendations. ${ }^{19}$ Whether more complex approaches to lead removal (eg, multivessel access ${ }^{33}$ ) will further reduce the proportion of open procedures remains speculative.

It may furthermore be argued that by applying our radical protocol, the resulting patient population may be less sick (higher proportion of mild disease). However, the relative proportions of isolated pocket infections as opposed to lead endocarditis $(\sim 65 / 35)$, the patient characteristics, and the baseline mortality figures suggest this is a typical and comparable ${ }^{6,26}$ patient cohort.

Major complications due to the interventional procedures were rare in this series, with no cases of cardiac tamponade or venous tears. There were 2 late tricuspid valve repairs $(1 \%)$. Damage to the valve cusps is not reported frequently, and laser-assisted extraction, which was not used for cost reasons, may offer an advantage over purely mechanical methods in this respect. ${ }^{34,35}$ Conversely, lethal complications during the extraction are predominantly known from laser-assisted extraction. Open procedures did not cause significant major morbidity.

Secondary wound explorations were performed in 11 cases $(5.7 \%)$, mainly for hematoma. All other wounds healed primarily, and only 2 local recurrences of infection were observed if all material had been removed. Three subclavian vein thromboses $(1.6 \%)$ occurred. Few studies have specifically reported "minor" complication rates in the treatment of device infection, but those reported for lead removal in general are usually higher than in this series. ${ }^{26,33}$

Radical debridement of subcutaneous tissue and fascial layers with subsequent extended suction drainage has permitted primary wound closure and proven highly effective in this series, but there are little data with which to compare.

\section{Eradication of Infection}

Published series demonstrate a high success rate of eradicating infection with slightly different approaches, more conservative patient selection, and a shorter minimum follow-up. ${ }^{2,26,33}$ Extraction of all prosthetic material is essential, because remnants, even parts of the outer lead insulation, caused recurrence in $71.4 \%$, which was suspected by others. ${ }^{2,24,26}$

Prolonged antibiotic treatment ${ }^{2}$ is thought to be important, but the data presented cannot prove this, and it is the practice in other institutions to interpose only days before reimplantation. ${ }^{19}$ It has been suggested that lead age and the extent of endocarditic involvement of the leads may change the requirement for prolonged antibiotic therapy. ${ }^{6,36}$ Recurrence in this series was independent of the type of infection (pocket/leads and bacterial strain), but in support of the former suggestion, it was associated with evidence of a chronic infectious process (previous lead dislocation and imminent cutaneous perforation) and the patient's dental status. The latter is a new finding; however, in analogy to infective endocarditis is not entirely surprising. ${ }^{6}$ The absence of prosthetic material in patients who did not receive another permanent device and a contralateral implantation site far from the old pocket as negative predictive factors for recurrence further support the argument. Thus, an absence of a source of bacteremias on the one hand and an absolute or relative absence of a target site on the other are expected to result in a lower reinfection rate. Again, this has not been specifically addressed in the literature but seems reasonable to conclude. 

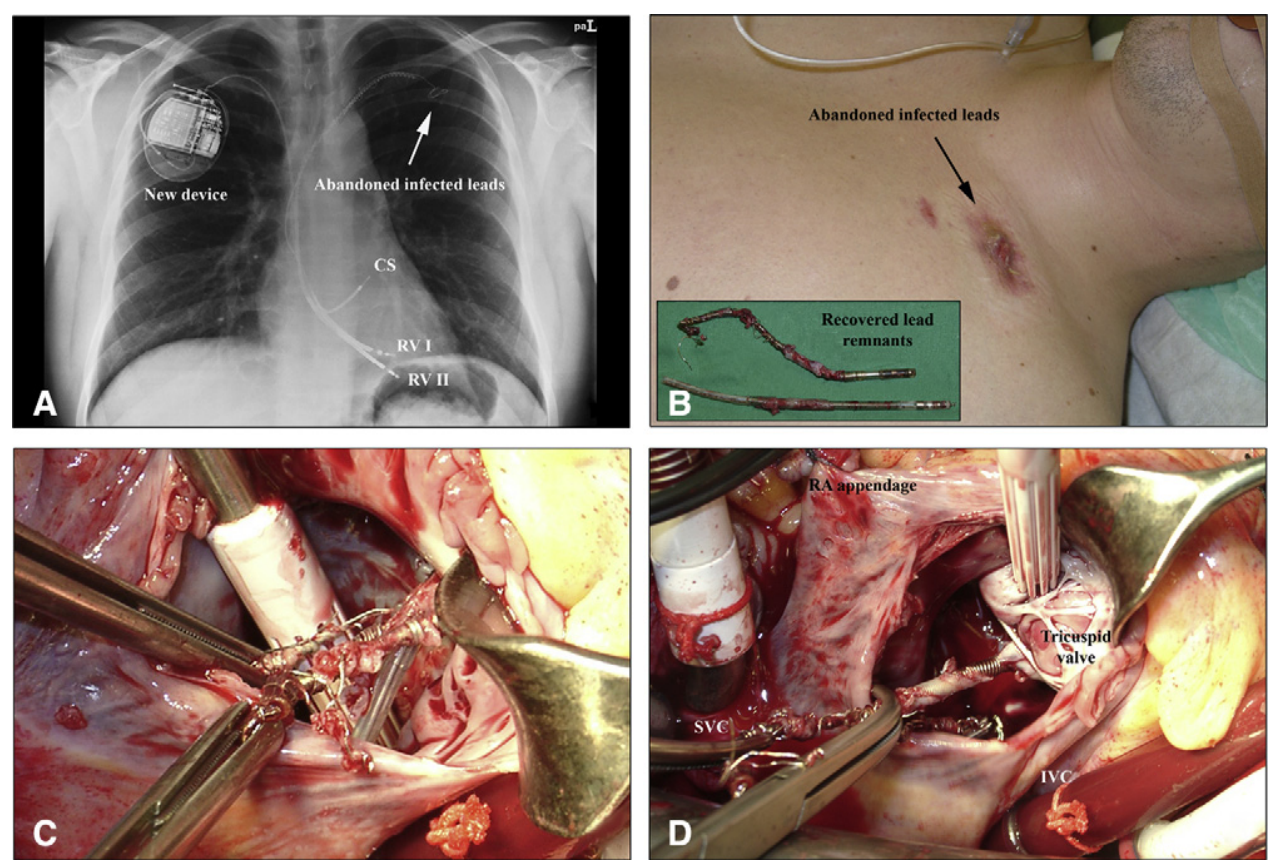

FIGURE 4. Persistent infection after limited explantation $(\mathrm{A}, \mathrm{B})$ in a patient. Intraoperative view from the patient's right (surgeon's view) into the right atrium showing the torn leads with vegetations (C) and the vicinity of the tricuspid valve (D). $C S$, Coronary sinus; $R V$, right ventricle; $R A$, right atrium; $S V C$, superior vena cava; $I V C$, inferior vena cava.

\section{Microbiology}

Positive culture results were observed in $80.6 \%$ of patients in whom the preoperative diagnosis of infection was unsecured, confirming that the clinical suspicion is usually justified. Also, the bacteria isolated were much as expected. ${ }^{36}$ No direct correlation was found between the strain and the failure to eradicate infection, although this had been expected according to the literature. ${ }^{6,37,38}$ This may be seen as supportive of a prolonged antibiotic regimen, despite the fact that it was not possible to show a direct correlation between the duration of interim antibiotic therapy and the success of eradication. Selection of resistance may be an issue in this case, ${ }^{36}$ but no evidence of recurrence with different, more resistant bacteria was found and only 3 cases of MRSA were observed in this series, in which 1 patient died of endocarditis. This may be an issue about changing the antibiotic prophylaxis in CIED surgery for the future, as has been proposed. ${ }^{19,36}$ The North American Society of Pacing and Electrophysiology/Heart Rhythm Society statement ${ }^{19}$ supports a radical approach but advocates a shorter period of interim antibiotic treatment without exactly specifying this (level of evidence C).

\section{Safety}

Device and lead removal has been shown to be safe in different patient cohorts. ${ }^{11,25,26,32,39}$ The details of the procedures, success rate, duration, $\mathrm{x}$-ray exposure, and use of adjuncts for lead extraction all compare favorably with the literature. ${ }^{25,26,32,33,40}$ Temporary pacing in outpatients proved safe, including fully dependent patients. This form of temporary pacing has been reported by others with favorable results. ${ }^{31}$ Hospital stay was 7 days for patients with a pacemaker and 12 days for patients with an ICD despite a median time to implantation of the new device of 6 weeks. Others have kept their patients as inpatients for the antibiotic treatment or because of a conventional temporary pacing system. ${ }^{2}$

Patients' acceptance of the staged procedure was good, with the majority reporting only minor difficulties with the external device. This has not been specifically addressed in the literature. Side effects of the antibiotic treatment dominated in this context.

\section{Limitations}

This was a single-center experience. General applicability of the results is thus limited. A bias may exist concerning the rate and severity of device infections because the referral rate of such patients to tertiary centers has changed considerably over the years. Thus, there remain a potential number of patients who receive conservative treatment and never present to the tertiary referral center. Conversely, some patients may die before the diagnosis is reached.

\section{CONCLUSIONS}

Protocol-driven, obligatory, complete removal of all prosthetic material in any CIED infection is safe and results 
in a successful eradication of infection in $98 \%$ of patients. Partial removal, previous lead dislocations, chronic pocket infection, and poor dental status are highly predictive for failure to eradicate infection. Interventional lead removal is well tolerated with acceptable low morbidity and mortality. Interim outpatient treatment of pacemakerdependent patients with a temporary, percutaneously externalized device is also safe and well tolerated by patients and health care facilities. In this series, neither old age nor significant concomitant disease has led to a significant increase of procedural risk.

\section{References}

1. Voigt A, Shalaby A, Saba S. Rising rates of cardiac rhythm management device infections in the United States: 1996 through 2003. J Am Coll Cardiol. 2006;48: 590-1.

2. Catanchin A, Murdock CJ, Athan E. Pacemaker infections: a 10-year experience. Heart Lung Circ. 2007;16:434-9.

3. Ferguson TB Jr, Ferguson CL, Crites K, Crimmins-Reda P. The additional hospital costs generated in the management of complications of pacemaker and defibrillator implantations. J Thorac Cardiovasc Surg. 1996;111: 742-51.

4. Klug D, Balde M, Pavin D, Hidden-Lucet F, Clementy J, Sadoul N, et al. Risk factors related to infections of implanted pacemakers and cardioverterdefibrillators: results of a large prospective study. Circulation. 2007;116: 1349-55.

5. Uslan DZ, Sohail MR, St Sauver JL, Friedman PA, Hayes DL, Stoner SM, et al. Permanent pacemaker and implantable cardioverter defibrillator infection: a population-based study. Arch Intern Med. 2007;167:669-75.

6. Baman TS, Gupta SK, Valle JA, Yamada E. Risk factors for mortality in patients with cardiac device-related infection. Circ Arrhythmia Electrophysiol. 2009;2: 129-34.

7. Chambers ST. Diagnosis and management of staphylococcal infections of pacemakers and cardiac defibrillators. Intern Med J. 2005;35(Suppl 2):S63-71.

8. Hemmer W, Frohlig G, Markewitz A. [Comment on NASPE recommendations for removal of permanently implanted, transvenous cardiac pacemaker and defibrillator electrodes]. Z Kardiol. 2002;91:956-68.

9. Klug D, Lacroix D, Savoye C, Goullard L, Grandmougin D, Hennequin JL, et al. Systemic infection related to endocarditis on pacemaker leads: clinical presentation and management. Circulation. 1997;95:2098-107.

10. Lo R, D'Anca M, Cohen T, Kerwin T. Incidence and prognosis of pacemaker lead-associated masses: a study of 1,569 transesophageal echocardiograms. J Invasive Cardiol. 2006;18:599-601.

11. Love CJ, Wilkoff BL, Byrd CL, Belott PH, Brinker JA, Fearnot NE, et al. Recommendations for extraction of chronically implanted transvenous pacing and defibrillator leads: indications, facilities, training. North American Society of Pacing and Electrophysiology Lead Extraction Conference Faculty. Pacing Clin Electrophysiol. 2000;23:544-51.

12. Sohail MR. Management of infected pacemakers and implantable cardioverterdefibrillators. Intern Med J. 2007;37:509-10.

13. Uslan DZ, Baddour LM. Cardiac device infections: getting to the heart of the matter. Curr Opin Infect Dis. 2006;19:345-8

14. Bloom H, Heeke B, Leon A, Mera F, Delurgio D, Beshai J, et al. Renal insufficiency and the risk of infection from pacemaker or defibrillator surgery. Pacing Clin Electrophysiol. 2006;29:142-5.

15. Dasgupta A, Montalvo J, Medendorp S, Lloyd-Jones DM, Ghossein C, Goldberger J, et al. Increased complication rates of cardiac rhythm management devices in ESRD patients. Am J Kidney Dis. 2007;49:656-63.

16. Sohail MR, Uslan DZ, Khan AH, Friedman PA, Hayes DL, Wilson WR, et al. Risk factor analysis of permanent pacemaker infection. Clin Infect Dis. 2007; 45:166-73.

17. Uslan DZ, Sohail MR, Friedman PA, Hayes DL, Wilson WR, Steckelberg JM, et al. Frequency of permanent pacemaker or implantable cardioverterdefibrillator infection in patients with gram-negative bacteremia. Clin Infect Dis. 2006;43:731-6.
18. Massoure PL, Reuter S, Lafitte S, Laborderie J, Bordachard P, Clementy J, et al. Pacemaker endocarditis: clinical features and management of 60 consecutive cases. Pacing Clin Electrophysiol. 2007;30:12-9.

19. Wilkoff BL, Love CJ, Byrd CL, Bongiorni MG, Carrillo RG, Crossley GH, et al. Transvenous lead extraction: Heart Rhythm Society Expert consensus on facilities, training, indications, and patient management: this document was endorsed by the American Heart Association (AHA). Heart Rhythm. 2009;6:1085-104.

20. Da CA, Kirkorian G, Cucherat M, Delahaye F, Chevalier P, Cerisier A, et al. Antibiotic prophylaxis for permanent pacemaker implantation: a meta-analysis. Circulation. 1998;97:1796-801.

21. Chang JP, Chen MC, Guo GB, Kao CL. Less-invasive surgical extraction of problematic or infected permanent transvenous pacemaker system. Ann Thorac Surg. 2005;79:1250-4.

22. Choo MH, Holmes DR, Gersh BJ, Maloney JD, Merideth J, Pluth JR, et al. Infected epicardial pacemaker systems. Partial versus total removal. $J$ Thorac Cardiovasc Surg. 1981;82:794-6.

23. Darouiche RO. Treatment of infections associated with surgical implants. $N$ Engl J Med. 2004;350:1422-9.

24. Griffet V, Caignault JR, Godon P, Guerard S, Brion R, Chevalier P. [Recurrent septicaemia following incomplete removal of pacemaker leads]. Presse Med. 2005;34:111-3.

25. Post JJ, Alexopoulos C, Fewtrell C, Giles R, Jones PD. Outcome after complete percutaneous removal of infected pacemaker systems and implantable cardiac defibrillators. Intern Med J. 2006;36:790-2.

26. Sohail MR, Uslan DZ, Khan AH, Friedman PA, Hayes DL, Wilson WR, et al. Management and outcome of permanent pacemaker and implantable cardioverter-defibrillator infections. J Am Coll Cardiol. 2007;49:1851-9.

27. Sohail MR, Uslan DZ, Khan AH, Friedmann PA, Hayes DL, Wilson WR, et al. Infective endocarditis complicating permanent pacemaker and implantable cardioverter-defibrillator infection. Mayo Clin Proc. 2008;83:46-53.

28. Kolker AR, Redstone JS, Tutela JP. Salvage of exposed implantable cardiac electrical devices and lead systems with pocket change and local flap coverage. Ann Plast Surg. 2007;59:26-30.

29. Klug D, Wallet F, Lacroix D, Marquie C, Kouakam C, Kacet S, et al. Local symptoms at the site of pacemaker implantation indicate latent systemic infection. Heart. 2004;90:882-6.

30. Dumont E, Camus C, Victor F, de Place C, Pavin D, Alonso C, et al. Suspected pacemaker or defibrillator transvenous lead infection. Prospective assessment of a TEE-guided therapeutic strategy. Eur Heart J. 2003;24:1779-87.

31. Braun MU, Rauwolf T, Bock M, Kappert U, Boscheri A, Schnabel A, et al. Percutaneous lead implantation connected to an external device in stimulationdependent patients with systemic infection-a prospective and controlled study. Pacing Clin Electrophysiol. 2006;29:875-9.

32. Rastan AJ, Doll N, Walther T, Mohr FW. Pacemaker dependent patients with device infection-a modified approach. Eur J Cardiothorac Surg. 2005;27:1116-8.

33. Bongiorni MG, Soldati E, Zucchelli G, Di Cori A, Segreti L, De Lucia R, et al. Transvenous removal of pacing and implantable cardiac defibrillating leads using single sheath mechanical dilatation and multiple venous approaches: high success rate and safety in more than 2000 leads. Eur Heart J. 2008;29:2886-93.

34. Hamid Sh, Arujna A, Khan S, Ladwiniec A, McPhail M, Bostock J, et al. Extraction of chronic pacemaker and defibrillator leads from the coronary sinus: laser infrequently used but required. Europace. 2009;11:213-5.

35. Jones SO, Eckart RE, Albert ChM, Epstein LM. Large, single-center, single operator experience with transvenous lead extraction: outcomes and changing indications. Heart Rhythm. 2008A;5:520-5.

36. Anselmino M, Vinci M, Comoglio Ch, Rinaldi M, Bongiorni MG, Trevi GP, et al. Bacteriology of infected extracted pacemaker and ICD leads. J Cardiovasc Med. 2009; 10:693-8.

37. Hudetz D, Ursic HS, Harris LG, Luginbuhl R, Friederich NF, Landmann R. Weak effect of metal type and ICA genes on staphylococcal infection of titanium and stainless steel implants. Clin Microbiol Infect. 2008;14:1135-45.

38. Klug D, Wallet F, Kacet S, Courcol RJ. Involvement of adherence and adhesion Staphylococcus epidermidis genes in pacemaker lead-associated infections. J Clin Microbiol. 2003;41:3348-50.

39. Calvagna GM, Evola R, Scardace G, Valsecchi S. Single operator experience with a mechanical approach for removal of pacing and implantable defibrillator leads. Europace. 2009;11:1505-9. Epub 2009 Aug 7.

40. Roux JF, Page P, Dubuc M, Thibault B, Guerra PG, Macle L, et al. Laser lead extraction: predictors of success and complications. Pacing Clin Electrophysiol. 2007;30:214-20. 